\title{
Identification Of A Novel Homozygous Intron 3 Splice Site (A>T) Mutation In The ARG1 Gene In Cerebral Palsy Pediatric Cases From Odisha, India
}

Chinmay Kumar Behera

Kalinga Institute of Medical Sciences

Amit Ranjan Rup

Kalinga Institute of Medical Sciences

Sagnika Samal

KIIT School of Biotechnology

Biswadeep Das ( $\square$ dviswadeep1983@gmail.com )

KIIT School of Biotechnology https://orcid.org/0000-0001-8214-3600

\section{Short Report}

Keywords: Hyperarginemia, pediatric case, novel mutation, whole genome sequencing.

Posted Date: March 7th, 2022

DOI: https://doi.org/10.21203/rs.3.rs-1402624/v1

License: (9) This work is licensed under a Creative Commons Attribution 4.0 International License. Read Full License 


\section{Abstract}

Background: Arginases are essential for the completion of the last step of the urea cycle. In hyperarginemia, an autosomal recessive disorder of the urea cycle, a regression of development occurs after the first year of life, followed by gradually progressive atonic cerebral palsy, spastic quadriplegia, and mental decline. ARG1 mutations have been reported in hyperarginemia in the Western population because they obliterated or partially damaged arginase activity. Hence, it is important to assess the ARG1 mutations in such cases of cerebral palsy with hyperarginemia in different populations.

Methods and Results: Two pediatric patients of two East Indian families presented with a range of manifestations including hypotonia of all limbs, mental retardation, and multiple episodes of seizure. The onset of the disease ranged from 1 to 3 years of age. Hyperammonemia (>250 micromoles) and serum hyperarginemia (> 350 micromoles) were observed in both patients. Whole-genome sequencing, followed by Sanger sequencing analysis of both patients confirmed the presence of a homozygous 3 ' splice site variation in intron 3 of the $A R G 1$ gene (chr6: g.131902357A>T) that affects the invariant AG acceptor splice site of exon 4 (c.330-2A>T; ENST00000356962.2).

Conclusion: The study reports the presence of a novel $A R G 1$ mutation in two different pediatric cases from Odisha, India associated with hyperarginemia. The pathogenicity of the mutation was robustly supported by the clinical phenotype, complete co-segregation with the disease, and biochemical observations.

\section{Background}

Arginase (EC 3.5.3.1) is a manganese-containing enzyme that belongs to a class of ureohydrolase. Larginine amidohydrolase is the systemic name of this enzyme that is involved in the catalysis of Larginine to L-ornithine and urea (Arginine $+\mathrm{H}_{2} \mathrm{O} \rightarrow$ Ornithine + Urea) [1]. This manganese metalloenzyme is the final enzyme of the urea cycle and is ubiquitous to all domains of life. The two main homeostatic purposes of the arginase activity are to remove ammonia from the body by urea synthesis and to produce ornithine, which is a precursor of polyamines, produced by ornithine decarboxylase (ODC) and are required for cell proliferation and regulation of multiple ion channels and prolines, produced by ornithine aminotransferase (OAT) is essential for collagen production.

The vertebrate contains two isoforms of arginase, named A1 (cytosolic arginase) and A2 (mitochondrial arginase) encoded by the $A R G 1$ and $A R G 2$ genes, respectively. A condition called arginase deficiency leads to a gradual build-up of ammonia and the amino acid arginine in the bloodstream. Protein breakdown generates ammonia, which is toxic when the levels become too high. Ammonia can cause serious problems to the nervous system. Arginase 1 predominates in $98 \%$ of the hepatic arginase activity. Its deficiency is a rare inherited disorder of the urea cycle characterized by complete or partial lack of the enzyme arginase in the liver and red blood cells and results in an autosomal-recessive (AR) inborn error of 
metabolism known as hyperarginemia. Approximately 1:350,000 to 1:1,000,000 people are diagnosed with hyperarginemia.

Hyperarginemia symptoms are rare during the neonatal period and rather appear during the age group of 2 to 4 years of age, and even in adults [2]. Patients typically suffer from developmental delay and regression of milestones, and if left untreated, this condition can develop gradually into spastic quadriparesis and intellectual disability. The condition can sometimes mimic cerebral palsy due to its non-progressive nature. Timely diagnosis, low protein diet, regimen for lowering ammonia, and supportive care are crucial [3]. In the early stages of childhood, affected children are frequently asymptomatic. Arginase is encoded by the Arg 1 gene present on $6 q 23.2$ of the human chromosome 6 . The pathogenic variants that cause hyperarginemia can occur in homozygous as well as heterozygous individuals, with probable correlation between genotype and phenotype. The first case in which the genetic basis of arginemia was characterized was a Japanese patient with compound heterozygous mutations [3].

\section{Index Case 1}

A four-year-old boy presented with a history of vomiting for the last four months and multiple episodes of generalized tonic-clonic convulsions (GTCS) over the past six days. The child vomited one to two episodes per day, non-projectile and non-bilious over the last four months. As a known case of seizure disorder, the child had the first episode of seizures at the age of three years old, which was of GTCS type without any fever-related symptoms, and was treated with syrup valproate. During this illness, he had convulsions, twice daily, lasting for 5 to 10 minutes, of GTCS - type, without fever for the past 6 days. This child was taken to a local hospital, where his valproate dose was increased and tablet clobazam was added. This child was born out of a third-degree consanguineous marriage, with non-significant antenatal and natal history, with normal vaginal delivery, having a birth weight of $3.200 \mathrm{~kg}$ with an uneventful neonatal period. He had a history of developmental delay with regression of milestones after the age of 3 years, as he could not stand or sit without support and assistance. Based on the National Immunization Schedule, the child had received all his vaccinations.

When admitted, the patient was irritable with a low Glasgow Coma Scale (GCS) of 14/15 with stable vitals. The child had no pallor, icterus, cyanosis, edema, lymphadenopathy, dysmorphism of the face, or neurocutaneous markers. Examinations of the central nervous system (CNS) revealed diminished interest in surroundings, hypotonia among muscles surrounding joints of the upper and lower limb, as well as the power of $3 / 5$ around both upper and lower limb joints. Bilateral deep tendon reflexes were absent, and bilateral plantar was extensor. All cranial nerves were intact. The sensory system, cerebellum, skull, and spine were normal. The rest of the system examination revealed no abnormality. From these history and examination findings, we treated the child as a case of an atonic type of cerebral palsy with a seizure disorder. Initial injections of sodium valproate were given and baseline investigations were sent.

On investigation, complete blood count $(\mathrm{CBC})$ was within the normal limit. The sepsis screen was negative. The liver function test, renal function test, thyroid profile, and serum electrolytes were normal. MRI brain revealed diffuse cerebral and cerebellar atrophy changes. EEG revealed focal epileptiform 
activity from the right frontotemporal region with secondary generalization. BERA was normal. Arterial blood gas (ABG) was normal with normal lactate levels. Serum ammonia level was $340 \mathrm{micromol} / \mathrm{liter}$ ( normal 11-35micromol/liter). As serum ammonia was high, inj. sodium valproate was stopped and inj. levetiracetam was started. A panel for Inborn Error of Metabolism was sent based on high serum ammonia, which revealed high arginine levels of 441 micromoles (Normal 3 - 130 micromoles) (Table 1). The child was put on an arginine-free diet and was treated with oral sodium benzoate at $250 \mathrm{mg} / \mathrm{kg} / \mathrm{day}$. After 48 hours of starting sodium benzoate, serum ammonia was decreased to $212 \mathrm{micromol} / \mathrm{liter}$. $\mathrm{He}$ was discharged with an arginine-free diet and sodium benzoate. A follow-up after 3 months revealed seizure-free behavior, no vomiting episodes, improved irritability, ability to stand without assistance, and decreased serum ammonia levels.

\section{Index Case 2}

A five-year-old girl presented with failure to thrive with multiple episodes of generalized tonic-clonic convulsions (GTCS) over the past five days. She was a known case of seizure disorder with the first episode of seizure that occurred at the age of two years old, which was of GTCS type and not associated with fever. The child was on syrup levetiracetam for the last 3years. The child was born out of nonconsanguineous marriage with a birth weight of $3 \mathrm{~kg}$ with an uneventful natal and postnatal history. There was a history of developmental delay with spasticity of both legs during walking after infancy without any regression of milestones.

When admitted, the child had a GCS of $15 / 15$ with stable vitals. The patient had mild pallor, but without any icterus, cyanosis, edema, lymphadenopathy, dysmorphism of the face, or neurocutaneous markers with evidence of malnutrition(weight $<3$ rd centile) Her head circumference was suggestive of microcephaly ( $<3^{\text {rd }}$ centile $)$ CNS examinations revealed a low level of mental function, hypertonia among all muscles surrounding all joints, as well as the power of $4 / 5$ around both upper and lower limb joints. Bilateral deep tendon reflexes were brisk, and bilateral plantar was extensor. All cranial nerves were intact. The sensory system, cerebellum, skull, and spine were normal. There were no extrapyramidal signs. Other system examinations revealed no abnormality. From these history and examination findings, we treated the child as a case of spastic type cerebral palsy with a seizure disorder. Initial injections of levetiracetam were given and baseline investigations were sent.

On investigation, $\mathrm{CBC}$ was within the normal limit except anemia, which was found to be a nutritional type on further evaluation. The sepsis screen was negative. The liver function test, renal function test, thyroid profile, and serum electrolytes were normal. MRI brain showed mild cerebral cortical atrophy. EEG revealed frontotemporal epileptic discharge. BERA was normal. Arterial blood gas (ABG) was normal with normal lactate levels. Serum ammonia level was $290 \mathrm{micromol} /$ liter (normal 11-35micromol/liter). A panel for Inborn Error of Metabolism was sent based on high serum ammonia, which revealed high arginine levels of 402 micromoles (Normal 3 - 130 micromoles) (Table 1). The child was put on an arginine-free diet and was treated with oral sodium benzoate at $250 \mathrm{mg} / \mathrm{kg} /$ day. After 48 hours of starting sodium benzoate, serum ammonia was decreased to $111 \mathrm{micromol} /$ liter. He was discharged with 
an arginine-free diet and sodium benzoate. A follow-up after 6 months revealed seizure-free behavior, ability to stand without assistance and walk with a normal gait, and decreased serum ammonia levels.

\section{Materials And Methods}

\section{DNA Extraction}

DNA was extracted from the blood of the two patients and one healthy control of the same age group, using DNeasy® DNA isolation kit (QIAGEN, USA) according to the manufacturer's protocol.

\section{Whole-Genome Sequencing}

Whole-genome sequencing (WGS) was conducted for the two patients and one healthy control. DNA extracted from blood was used to perform targeted gene capture using a custom capture kit. The libraries were sequenced to mean $>80-100 \mathrm{X}$ coverage on the Illumina sequencing platform. The GATK best practices framework was followed for the identification of variants in the sample using Sentieon (v201808.01). The sequences obtained were aligned to the human reference genome (GRCh37/hg19) using Sentieon aligner and analyzed using Sentieon for removing duplicates, recalibration, and realignment of indels. Sentieon haplotype caller has been used to identify variants that are relevant to the clinical indication. Gene annotation of the variants is performed using the VEP program against the Ensembl release 99 human gene model. In addition to SNVs and small Indels, copy number variants (CNVs) are detected from targeted sequence data using the ExomeDepth (v1.1.10) method. This algorithm detects rare CNVs based on a comparison of the read-depths of the test data with the matched aggregate reference dataset. Clinically relevant mutations were annotated using published variants in literature and a set of diseases databases - ClinVar, OMIM (updated on 11th May 2020), GWAS, HGMD (v2020.2), and SwissVar. Common variants are filtered based on allele frequency in 1000 Genome Phase 3, gnomAD (v2.1), EVS, dbSNP (v151), 1000 Japanese Genomes, and our internal Indian population database. Non-synonymous variant effects were effect calculated using multiple algorithms such as PolyPhen-2, SIFT, MutationTaster2, and LRT. Only non-synonymous and splice site variants found in the newborn screening panel genes were used for clinical interpretation. Silent variations that do not result in any change in amino acid in the coding region were not reported. Both probability-based and qualitybased algorithms were used to identify single nucleotide variants (SNVs) and insertions/deletions (Indels). For gene/variant prioritization, a minimum depth of $30 x$ and $1 \%$ as the minor allele frequency cut-off was selected.

\section{Sanger Sequencing}

To confirm the presence of the mutation at the specific locus, Sanger sequencing was performed for the two patients and one healthy control using the BIGDYE chemistry on an ABI3730 sequencer (Applied Biosystems $\left.{ }^{\circledR}\right)$. The sequences were further visualized and analyzed using Chromas Lite ${ }^{\circledR}$ software (Technelysium ${ }^{\circledR}$, South Brisbane, QLD, Australia) and Seqscape ${ }^{\circledR}$ (Applied Biosystems ${ }^{\circledR}$ ). 


\section{Results And Discussion}

Arginemia represents a different clinical presentation than other enzymatic defects of the urea cycle. Children with an arginase 1 deficiency can develop devastating neuropsychiatric problems; however, the condition is potentially curable [4]. Terheggen et al. described the association of arginase deficiency and hyperarginemia [5]. Patients with arginemia typically have normal neonatal and early childhood periods; however, after the age 1 to 3 , linear growth may be retarded. Common clinical presentations are spastic diplegia, seizures, intermittent ataxia, deteriorating cognitive function, hyperactivity, mental retardation, and choreoathetosis [6]. Symptomatic hyperammonemia leading to coma is uncommon. Neurologic manifestation is mostly linked to hyperarginemia; however, the neuropathogenic mechanism is not known. Hyperarginemia may mimic cerebral palsy [7], which is one of the few treatable IEM. In arginase deficiency, laboratory findings reveal hyperammonemia and hyperarginemia. With a delay in diagnosis and management, there is increase of CNS manifestations, spasticity, in addition to loss of ambulation and complete loss of bladder and bowel. For the prevention of neurological deterioration, protein intake should be restricted and essential amino acids to be supplemented [8]. Diagnosis was confirmed by the identification of biallelic pathogenic variants in $A R G 1$ [9]. A whole-genome sequencing study performed on both patients and healthy control of the same age group confirmed the presence of a homozygous $3^{\prime}$ splice site variation in intron 3 of the ARG1 gene (chr6: g.131902357A>T; Depth: 99x) that affects the invariant AG acceptor splice site of exon 4 (c.330-2A>T; ENST00000356962.2) (Fig 1), and the variant was predicted to be pathogenic. Management of hyperarginemia involves lowering arginine levels and to avoid hyperammonemic episodes by the use of sodium benzoate, which is a nitrogen scavenging therapy. To treat and prevent hyperammonemia in the urea cycle disorder, sodium benzoate therapy is safe and most effective [10]. These patients are less prone to hyperammonemic episodes and mostly asymptomatic in newborn life; hence, diagnosing such cases become late. Because arginemia is a treatable IEM, its identification is crucial, and so newborn genetic screening could be an effective method for the early diagnosis of hyperarginemia using genome sequencing and biochemical approaches.

\section{Declarations}

\section{Funding}

The authors declare that no funds, grants, or other support were received during the preparation of this manuscript.

\section{Competing Interests}

The authors have no relevant financial or non-financial interests to disclose.

\section{Author Contributions}

All authors contributed to the study conception and design. Material preparation, data collection and analysis were performed by $C K B, A R R$, SS and BD. The final draft of the manuscript was written by $C K B$, 
$A R R$ and $B D$ and all authors commented on previous versions of the manuscript. All authors read and approved the final manuscript.

\section{Ethics approval}

This study was performed and approved by the Institute Ethical Committee of KIIT School of Biotechnology, KIIT Deemed to be University, Bhubaneswar.

\section{Consent to participate}

Written informed consent was obtained from the parents.

\section{References}

1. Brusilow SW (2001). Urea cycle enzymes. The metabolic and molecular bases of inherited disease 1909-64.

2. Scaglia F, Lee B (2006). Clinical, biochemical, and molecular spectrum of hyperarginemia due to arginase I deficiency. In American Journal of Medical Genetics Part C: Seminars in Medical Genetics (Vol. 142, No. 2, pp. 113-120). Hoboken: Wiley Subscription Services, Inc., A Wiley Company.

3. Carvalho DR, Brum JM, Speck-Martins CE, Ventura FD, Navarro MM, Coelho KE, Portugal D, Pratesi $R$ (2012). Clinical features and neurologic progression of hyperarginemia. Pediatric neurology 46(6):369-74.

4. Bachmann C (2003). Outcome and survival of 88 patients with urea cycle disorders: a retrospective evaluation. Eur J Pediatr 162:410- 6. doi: 10.1007/s00431-003-1188-9

5. Terheggen HG, Schwenk A, Lowenthal A, Van Sande M, Colombo JP (1969). Argininaemia with arginase deficiency. Lancet ;2:748-9.

6. Leelavathi V, Shivaprasad SM, Naveen R (2013). Clinical Case Report on Arginase Deficiency: An Unusual Indication for Hemodialysis. International Journal of Contemporary Medicine 1(2):24.

7. Ustkoyuncu PS, Kendirci M, Gokay S, Kardas F, Gumus H, Per H, Poyrazoglu HG, Bayram AK, Canpolat M, Kumandas S (2020). A Rare Cause of Spasticity and Microcephaly: Arginemia. Turk J Neurol 26:250-2.

8. Lee BH, Jin HY, Kim GH, Choi JH, Yoo HW (2011). Arginemia presenting with progressive spastic diplegia. Pediatric neurology 44(3):218-20.

9. Sun A, Crombez EA, Wong D. Arginase Deficiency Synonyms: ARG1 Deficiency, Arginase-1 Deficiency, Hyperarginemia.

10. Husson MC, Schiff M, Fouilhoux A, Cano A, Dobbelaere D, Brassier A, Mention K, Arnoux JB, Feillet F, Chabrol B, Guffon N (2016). Efficacy and safety of iv sodium benzoate in urea cycle disorders: a multicentre retrospective study. Orphanet journal of rare diseases 11(1):1-8.

\section{Table}


Table 1 Clinical data of two different pediatric cases associated with hyperarginemia due to a mutation in the $A R G 1$ gene. 


\begin{tabular}{|c|c|c|}
\hline Mutated gene & ARG1 & \\
\hline Gender & M & $\mathrm{F}$ \\
\hline Clinical diagnosis & $\begin{array}{l}\text { Atonic type of cerebral palsy } \\
\text { with seizure disorder }\end{array}$ & $\begin{array}{l}\text { Spastic Cerebral palsy with seizure } \\
\text { disorder with failure to thrive(FTT) }\end{array}$ \\
\hline $\begin{array}{l}\text { Motor symptoms } \\
\text { onset age }\end{array}$ & 3 years & 2 years \\
\hline $\begin{array}{l}\text { Initial examination } \\
\text { age }\end{array}$ & 4 years & 5 years \\
\hline UL \& LL Tones & Hypotonia & Hypertonia \\
\hline UL \& UL Power & $3 / 5$ & $4 / 5$ \\
\hline Tendon reflexes & Absent & Increased \\
\hline Plantar response & $\uparrow$ & $\uparrow$ \\
\hline GCS & $14 / 15$ & $15 / 15$ \\
\hline Cerebellar signs & Absent & Absent \\
\hline $\begin{array}{l}\text { DUL/DLL } \\
\text { muscle atrophy }\end{array}$ & $-/+$ & $+/+$ \\
\hline Facial atrophy & - & - \\
\hline $\begin{array}{l}\text { Cognitive/psychiatric } \\
\text { Signs }\end{array}$ & $+/+$ & $+/+$ \\
\hline Sensory loss & - & - \\
\hline Optic atrophy & - & - \\
\hline Extrapyramidal signs & - & - \\
\hline Other signs & Anal incontinence & Severe urinary/anal incontinence \\
\hline Brain MRI & $\begin{array}{l}\text { Diffuse cerebral and } \\
\text { cerebellar atrophy }\end{array}$ & Mild cerebral cortical atrophy \\
\hline $\begin{array}{l}\text { Plasma amino acid } \\
\text { levels }\end{array}$ & $\uparrow$ Arginine & $\uparrow$ Arginine \\
\hline Serum ammonia & $+++/+++$ & $+++/+++$ \\
\hline Summary & Hypotonia, & Hypertonia, \\
\hline
\end{tabular}




$\begin{array}{ll}\text { cog/psych symptoms, } & \text { cog/psych symptoms, } \\ \text { seizure, } & \text { seizure, sphincter disturbances } \\ \text { sphincter disturbances } & \end{array}$

Extensor planter response: $\uparrow$ Unilateral, $\uparrow \uparrow$ Bilateral, $\leftrightarrow$ Mute, $\downarrow$ Flexor.

[1] UL, upper limbs; LL, lower limbs; PUL/PLL, proximal upper/lower limbs; DUL/DLL, distal upper/lower limbs; Cog./psych., cognitive/psychiatric.

Clinical signs severity: - Absent, + Mild, ++ Moderate, +++ Severe. Tendon reflexes: $\uparrow$ Increased tendon reflex.

\section{Figures}

\section{Figure 1}

Position of the homozygous 3' splice site variation in intron 3 of the ARG1 gene (chr6: g.131902357A>T) that affects the invariant AG acceptor splice site of exon 4 (c.330-2A>T; ENST00000356962.2)and the protein. 